\title{
Stepwise development of robotic donor right hepatectomy according to the anatomical variations in the hilum and the graft volume
}

\author{
Hyeyeon Yang, Gihong Choi
}

Department of Surgery-Hepatobiliary, Severance Hospital, Seoul, Korea

Background: Initial strict selections of donor without anatomical variations are recommended for minimally invasive living donor liver transplantation (LDLT) program because the donor safety is the most paramount. In this study, we introduced our stepwise development of robotic donor LDLT from donors with from favorable to unfavorable anatomies.

Methods: From April 2016 to October 2020, 80 donors received robotic donor right hepatectomy. All donors were divided according to the variations of the portal vein and bile duct and the graft volume $(>800 \mathrm{~mL})$. Donors who had at least one variable that satisfy beyond the three extended criteria were defined as 'unfavorable group.' The proportion of variations was analyzed according to the four periods and perioperative outcomes were compared between favorable and unfavorable group.

Results: Among 80 cases, portal vein variation and bile duct variation were observed 10 cases and 22 cases, respectively. Donors who had graft weight more than $800 \mathrm{~g}$ were 22 cases. Unfavorable group donors were eight cases in first and second period, respectively. In third period, nine donors were unfavorable group. In recent 20 cases, 14 donors were unfavorable group. Comparing the perioperative outcomes between favorable and unfavorable group, there were no significant differences regarding total operative time, warm ischemic time, estimated blood loss and postoperative complication.

Conclusions: Stepwise development of robotic donor right hepatectomy showed comparable perioperative outcomes in donor with the anatomical variations in the hilum and larger graft volume and seems to a reasonable way for a safe and successful minimally invasive LDLT program.

Corresponding author: Gihong Choi

E-mail:CHOIGH@yuhs.ac

() The Korean Society for Transplantation

This is an Open Access article distributed under the terms of the Creative Commons Attribution Non-Commercial License (http://creativecommons.org/licenses/by-nc/4.0/) which permits unrestricted non-commercial use, distribution, and reproduction in any medium, provided the original work is properly cited. 